\title{
Behavioral Analysis of Cooling Tower in Steam Turbine Power Plant using Reliability, Availability, Maintainability and Dependability Investigation
}

\author{
Nivedita Gupta, Monika Saini* and Ashish Kumar \\ Department of Mathematics \& Statistics, Manipal University Jaipur, Jaipur (Rajasthan) - 303007
}

Received 26 November 2019; Accepted 26 February 2020

\begin{abstract}
The main objective of present study is to provide a novel technique for identification of most critical component of cooling tower in steam turbine power plant. In steam turbine power plants various subsystems works in a series structure out of which cooling tower plays a prominent role in operation of plant. Cooling tower is comprised using six components in series structure. In present analysis, reliability, availability, maintainability and dependability (RAMD) approach has been utilized to find the performance measures of the cooling tower. Markov birth death process has been used to develop mathematical models for each component of cooling tower. Chapman Kolmogorov differential equations for each component has been formulated. All failure and repair time random variables follow exponential distribution, and all are statistically independent. Sufficient repair facility always remains with system. The numerical results for reliability, maintainability, dependability and steady state availability for different components of cooling tower have been derived. Other measures such as mean time to failure (MTTF), mean time to repair (MTTR) and dependability ratio, which help us to predict system performance has also been calculated. Numerical analysis reflects that availability of the system is 0.9775468 , reliability of the system after 10 months is 0.703280 and become 0.085094 after 70 months. Maintainability of the system is 0.997239 and Dependability is 0.977985 . Through, the derived numerical results operational performance of cooling tower has been assessed and it is recommended that findings are very useful for designers and maintenance engineers of cooling tower.
\end{abstract}

Keywords: Cooling tower, Markov Birth Death process, Reliability, Maintainability, Availability, Dependability, Mean time to failure and Mean time to repair.

\section{Introduction}

Condensers use relatively cool water to condense the steam from the turbines by different means. This decreases back pressure, which in turn reduces the consumption of steam and thus the consumption of gas, while at the same time increasing energy and recycling boiler water. However, the condensers need ample supply of cooling water without which they are inefficient. Then the role of cooling tower came into existence. A cooling tower is a heat-rejection machine that by cooling a water flow to a lower temperature rejects waste heat to the atmosphere. Smooth running of power plant also depends on smooth running of it as well. So, it is important to increase its reliability and availability and require adequate strategies to find its critical component and time to time proper maintenance of it. As a matter of fact, every system is always built to fulfill and justify its operational requirement, which is guaranteed by the system's reliability and time-totime availability. Reliability technology provides designers a structure to establish a proper system design, optimize its operating characteristics and formulate maintenance policies. System designers need to be aware about the most critical component and its time-to-time maintenance for this reason. Reliability, availability, maintainability and dependability (RAMD) is one of the methods to help in achieving this goal. This helps to identify the most critical component and to

\footnotetext{
*E-mail address: drmnksaini4@gmail.com

ISSN: $1791-2377$ @ 2020 School of Science, HHU. All rights reserved.

doi:10.25103/|iestr.132.23
}

ensure that they have proper maintenance policies. Certain parameters such as performance, servicing, MTTR, MTTF, Dependability ratio are also measured.

Many researchers tried to analyze the reliability and maintainability of various industrial systes. Using WeibullMarkov stochastic method, Casteren et al. [1] performed performance tests in electrical power systems. Eti et al. [2] surveyed the output of gas turbine plants in Nigeria's A-fam power plant. Arora and Kumar [3] presented a case study to optimize resource allocation and benefit in the thermal power plant coal handling process through complex programming and operational evaluation to increase the efficiency of the system. Carazas et al. [4] proposed a methodology for the assessment of gas turbine power plant for performance and availability analysis based on the concept of process reliability. Carazas et al. [5] provided a performance and availability evaluation system for HRSGs built in a combined cycle power plant. Kumar et al. [6] addressed the use of genetic algorithms to maximize the quality of a fertilizer plant's CO shift conversion process. Adhikarya et al. [7] suggested a comparative study focused on efficiency, stability and availability of two units of a coal-fired thermal power station in eastern India. Obeidat et al. [8] evaluate each unit's actions at $\mathrm{AL}$ - Hussein thermal power station and establish efficient plant maintenance strategies. Aggarwal et al. [9] addressed the reliability evaluation of a fertilizer plant's urea synthesis process using a Markovian method. For various choices of system subsystem failure and repair rates long-run availability, reliability and mean time between failures have been calculated. Corvaro et al. [10] developed the technique 
for evaluating reciprocating compressor output with the aid of efficiency, availability, maintenance (RAM) and taking failure and repair rates as distributed exponentially. Tsarouhas [11] analyzed the quality of the wine packaging production line using the RAMD methodology and derives various estimates of system performance and identified the best suited distribution. Recently, Saini et al. [12] and Goyal et al. [13] used RAMD methodology to identify most sensitive component of serial processes like evaporation system in the sugar industry and water treatment plant. In this study, the performance indices of the power generating system through STP have been broken down. For analyze the power system, basic principles of probability theory and Markovian birth-death process have been used. In a Markov process, as the process moves from one stage to the next, the probability of its moving from a particular state ' $i$ ' to another state ' $j$ ' is independent of how the process arrived at state ' $i$ ' in the first place. The system follows the memoryless property. The paper consists of four sections, including present introductory section. System description, and assumptions are explained in section second. RAMD analysis is performed in section 3 . Finally, section 4 is devoted to conclusion and implication of the results.

\section{System description and assumptions}

\section{(i) System description}

In this section, a brief description of cooling tower in steam turbine power plant has been given. Cooling tower mainly consists of seven components namely hydro turbine, hydraulic valves, water spray system, automatic deaerator valves, cooling water pump, motor valves and standpipe. All components are arranged in series configuration. The pictorial representation of components is appended in fig. 1 .

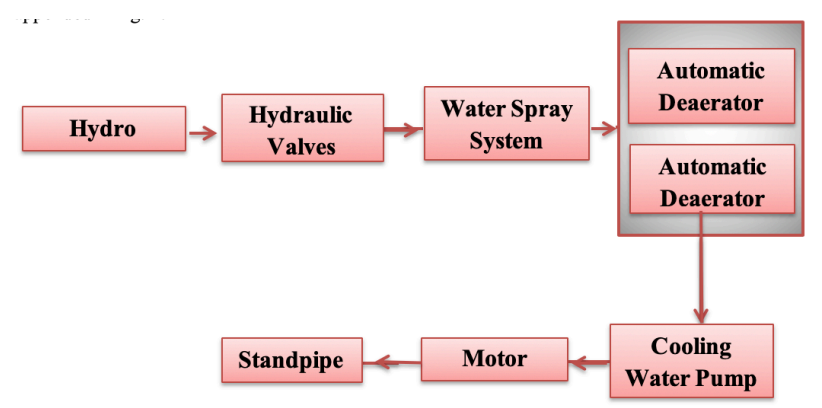

Fig. 1. Configuration Diagram of Cooling Tower Subsystem

\section{a) Subsystem A (Hydro Turbine)}

It consists of one unit of hydro turbine. This unit's failure causes complete system failure as it is connected to the following unit in sequence.

b) Subsystem B (Hydraulic Valves)

It consists of one set of hydraulic valves. This unit's failure causes complete system failure as it is connected to the following unit in sequence.

\section{c) Subsystem C (Water Spray System)}

It consists of one unit of water spray system. This unit's failure causes complete system failure as it is connected to the following unit in sequence.

\section{d) Subsystem D (Automatic Deaerator Valves)}

It consists of two sets of automatic deaerator valves; one is operative and other is in cold standby. The failure rate of both the units are same and failure of both units tends to system failure. e) Subsystem E (Cooling Water Pump)

It consists of one unit of Cooling water Pump. This unit's failure causes complete system failure as it is connected to the following unit in sequence.

\section{f) Subsystem F (Motor Valves)}

It consists of one set of motor valves. This unit's failure causes complete system failure as it is connected to the following unit in sequence.

\section{g) Subsystem G (Stand Pipe)}

It consists of one set of motor valves. This unit's failure causes complete system failure as it is connected to the following unit in sequence.

\section{(ii) Assumptions}

- The failure rates and repair rates of each subsystem follows exponential distribution.

- $\quad$ The failure and repair rates are statistically independent to each other.

- There are no simultaneous failures among the subsystem.

- There are enough repair and replacement facilities. Repairmen always present in plant and performance wise repaired system is as good as new.

- The switchover devices used for standby subsystems are perfect.

\section{RAMD analysis of the system}

By considering Markov birth death process for mathematical modeling of the cooling tower Chapman Kolmogorov differential equations for each of the sub-systems have been derived. All failure and repair rates of each subsystem has been considered as constant as shown in table 1. For each subsystem, a state transition diagram has been formulated. In each subsystem, by solving corresponding ChapmanKolmogorov differential equations in a steady state and simultaneously using normalizing conditions, system performance measures such as maintainability, availability, reliability, mean time to failure (MTTF), mean time to repair (MTTR) and reliability ratio has been derived.

Table 1. Failure and Repair rates of components of cooling tower

\begin{tabular}{c|c|c}
\hline Subsystem & Failure Rates $(\boldsymbol{\beta})$ & Repair rates $(\boldsymbol{\mu})$ \\
\hline $\mathrm{S}_{1}$ & $\beta_{1}=0.003$ & $\mu_{1}=0.42$ \\
$\mathrm{~S}_{2}$ & $\beta_{2}=0.0073$ & $\mu_{2}=1.25$ \\
$\mathrm{~S}_{3}$ & $\beta_{3}=0.0009$ & $\mu_{3}=0.09$ \\
$\mathrm{~S}_{4}$ & $\beta_{4}=0.006$ & $\mu_{4}=0.52$ \\
$\mathrm{~S}_{5}$ & $\beta_{5}=0.0025$ & $\mu_{5}=0.18$ \\
$\mathrm{~S}_{6}$ & $\beta_{6}=0.005$ & $\mu_{6}=0.95$ \\
$\mathrm{~S}_{7}$ & $\beta_{7}=0.0045$ & $\mu_{7}=0.75$ \\
\hline
\end{tabular}

The RAMD indices for subsystems of cooling tower of steam turbine power plant (STPP) are computed as

\section{a) RAMD indices for subsystem $S_{\mathbf{1}}$}

This subsystem has single unit only. Failure of it leads to complete system failure. The transition diagram and Chapman - Kolmogorov differential equations associated with it is given as: 


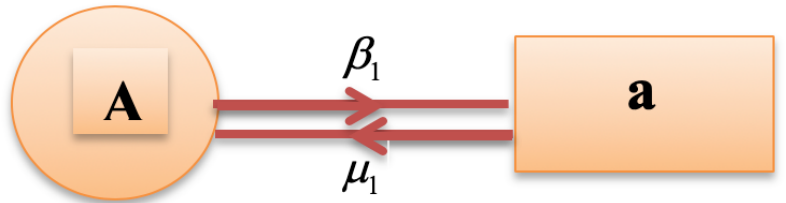

Fig. 2. Transition diagram of hydro turbine

$P_{0}^{\prime}(t)=-\beta_{1} P_{0}(t)+\mu_{1} P_{1}(t)$

$P_{1}^{\prime}(t)=\beta_{1} P_{0}(t)-\mu_{1} P_{1}(t)$

Under steady state, equation 9 and 10 reduces to

$P_{1}=\frac{\beta_{1}}{\mu_{1}} P_{0}$

Now, using normalization condition

$P_{0}+P_{1}=1 \Rightarrow P_{0}+\frac{\beta_{1}}{\mu_{1}} P_{0}=1 \Rightarrow P_{0}=\frac{\mu_{1}}{\mu_{1}+\beta_{1}}$

Now, by using equations (Appendix 1-5, 7-8 \& 4) important system performance measures have been derived and appended in table -4 .

\section{b) RAMD indices for subsystem $\mathbf{S}_{\mathbf{2}}$}

This subsystem has single unit only. Failure of it leads to complete system failure. The transition diagram and Chapman - Kolmogorov differential equations associated with it is given as:

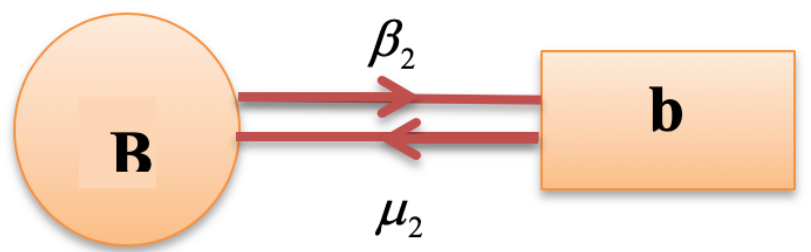

Fig. 2. Transition diagram of hydraulic valves

$$
P_{0}^{\prime}(t)=-\beta_{2} P_{0}(t)+\mu_{2} P_{1}(t)
$$

$P_{1}^{\prime}(t)=\beta_{2} P_{0}(t)-\mu_{2} P_{1}(t)$

Under steady state, equation 13 and 14 reduces to

$P_{1}=\frac{\beta_{2}}{\mu_{2}} P_{0}$

Now, using normalization condition

$P_{0}+P_{1}=1 \Rightarrow P_{0}+\frac{\beta_{2}}{\mu_{2}} P_{0}=1 \Rightarrow P_{0}=\frac{\mu_{2}}{\mu_{2}+\beta_{2}}$

Now, by using equations (1-5, 7-8 \& 16) important system performance measures have been derived and appended in table 4. c) RAMD indices for subsystem $S_{3}$

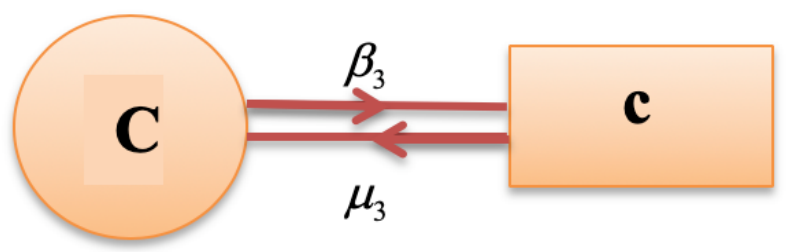

Fig. 3. Transition diagram of water spray system

This subsystem has single unit only. Failure of it leads to complete system failure. The transition diagram and Chapman - Kolmogorov differential equations associated with it is given as:

$$
P_{0}^{\prime}(t)=-\beta_{3} P_{0}(t)+\mu_{3} P_{1}(t)
$$

$P_{1}^{\prime}(t)=\beta_{3} P_{0}(t)-\mu_{3} P_{1}(t)$

Under steady state, equation 17 and 18 reduces to

$P_{1}=\frac{\beta_{3}}{\mu_{3}} P_{0}$

Now, using normalization condition

$P_{0}+P_{1}=1 \Rightarrow P_{0}+\frac{\beta_{3}}{\mu_{3}} P_{0}=1 \Rightarrow P_{0}=\frac{\mu_{3}}{\mu_{3}+\beta_{3}}$

Now, by using equations (Appendix 1-5, 7-8 \& 12) important system performance measures have been derived and appended in table 4.

d) RAMD indices for subsystem $\mathbf{S}_{4}$

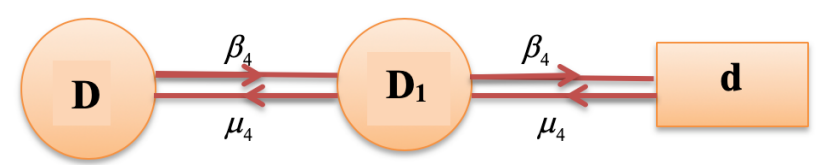

Fig. .4. Transition diagram of automatic deaerator valves

This subsystem has single unit working at a time only but with one cold standby unit. Failure of both leads to complete system failure. The transition diagram and Chapman Kolmogorov differential equations associated with it are given as:

$P_{0}^{\prime}(t)=-\beta_{4} P_{0}(t)+\mu_{4} P_{1}(t)$

$P_{1}^{\prime}(t)=\beta_{4} P_{0}(t)-\left(\beta_{4}+\mu_{4}\right) P_{1}(t)+\mu_{4} P_{2}(t)$

$P_{2}^{\prime}(t)=\beta_{4} P_{1}(t)-\mu_{4} P_{2}(t)$

Under steady state, equation 21,22 and 23 reduces to

$P_{1}=\frac{\beta_{4}}{\mu_{4}} P_{0}$ 
$P_{2}=\frac{\beta_{4}^{2}}{\mu_{4}^{2}} P_{0}$

Now, using normalization condition:

$P_{0}+P_{1}+P_{2}=1 \Rightarrow P_{0}+\frac{\beta_{6}}{\mu_{6}} P_{0}+\frac{\beta_{6}^{2}}{\mu_{6}^{2}} P_{0}=1 \Rightarrow$

$P_{0}=\left(1+\frac{\beta_{6}}{\mu_{6}}+\frac{\beta_{6}^{2}}{\mu_{6}^{2}}\right)^{-1}$

Now, by using equations (Appendix 1-5, 7-8 \& 18) important system performance measures have been derived and appended in table 4.

\section{e) RAMD indices for subsystem $S_{5}$}

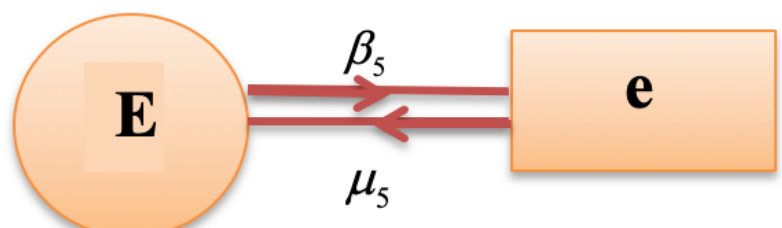

Fig. 5. Transition diagram of cooling water pump

This subsystem has single unit only. Failure of it leads to complete system failure. The transition diagram and Chapman - Kolmogorov differential equations associated with it is given as:

$$
\begin{aligned}
& P_{0}^{\prime}(t)=-\beta_{5} P_{0}(t)+\mu_{5} P_{1}(t) \\
& P_{1}^{\prime}(t)=\beta_{5} P_{0}(t)-\mu_{5} P_{1}(t)
\end{aligned}
$$

Under steady state, equation 27 and 28 reduces to

$P_{1}=\frac{\beta_{5}}{\mu_{5}} P_{0}$

Now, using normalization condition

$P_{0}+P_{1}=1 \Rightarrow P_{0}+\frac{\beta_{5}}{\mu_{5}} P_{0}=1 \Rightarrow P_{0}=\frac{\mu_{5}}{\mu_{5}+\beta_{5}}$

Now, by using equations $(1-5,7-8 \& 30)$ important system performance measures have been derived and appended in table 4

f) RAMD indices for subsystem $\mathbf{S}_{6}$

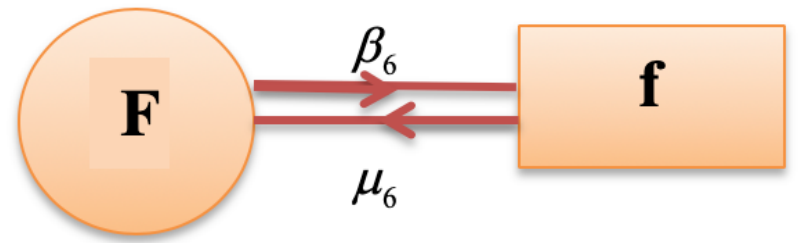

Fig. 6. Transition diagram of motor valves
This subsystem has single unit only. Failure of it leads to complete system failure. The transition diagram and Chapman - Kolmogorov differential equations associated with it is given as:

$P_{0}^{\prime}(t)=-\beta_{6} P_{0}(t)+\mu_{6} P_{1}(t)$

$P_{1}^{\prime}(t)=\beta_{6} P_{0}(t)-\mu_{6} P_{1}(t)$

Under steady state, equation 23 and 24 reduces to

$P_{1}=\frac{\beta_{6}}{\mu_{6}} P_{0}$

Now, using normalization condition

$P_{0}+P_{1}=1 \Rightarrow P_{0}+\frac{\beta_{6}}{\mu_{6}} P_{0}=1 \Rightarrow P_{0}=\frac{\mu_{6}}{\mu_{6}+\beta_{6}}$

Now, by using equations (1-5, 7-8 \& 34) important system performance measures have been derived and appended in table 4 .

\section{g) RAMD indices for subsystem $\mathbf{S}_{7}$}

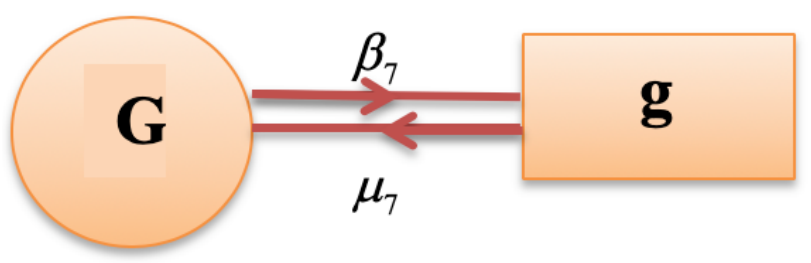

Fig. 7. Transition diagram of stand pipe

This subsystem has single unit only. Failure of it leads to complete system failure. The transition diagram and Chapman - Kolmogorov differential equations associated with it is given as:

$P_{0}^{\prime}(t)=-\beta_{7} P_{0}(t)+\mu_{7} P_{1}(t)$

$P_{1}^{\prime}(t)=\beta_{7} P_{0}(t)-\mu_{7} P_{1}(t)$

Under steady state, equation 27 and 28 reduces to

$P_{1}=\frac{\beta_{7}}{\mu_{7}} P_{0}$

Now, using normalization condition

$P_{0}+P_{1}=1 \Rightarrow P_{0}+\frac{\beta_{7}}{\mu_{7}} P_{0}=1 \Rightarrow P_{0}=\frac{\mu_{7}}{\mu_{7}+\beta_{7}}$

Now, by using equations (Appendix 1-5, 7-8 \& 30) important system performance measures have been derived and appended in table 4. 


\section{System reliability}

All seven subsystems are connected through one another in sequence. Just one failure leads to complete failure of the system. The overall system reliability of the cooling tower is determined by

$$
\begin{aligned}
& R_{S y s}(t)=R_{S_{1}}(t) * R_{S_{2}}(t) * R_{S_{3}}(t) * R_{S_{4}}(t) * R_{S_{5}}(t) * R_{S_{6}}(t) * R_{S_{7}}(t) \\
& \quad=e^{-\left(\beta_{1}+\beta_{2}+\beta_{3}+2 \beta_{4}+\beta_{5}+\beta_{6}+\beta_{7}\right) t} \\
& \Rightarrow R_{S y s}(t)=e^{-0.0352 t}
\end{aligned}
$$

The variation in reliability with respect to different time instant is compiled in table 2

Table 2. Variation of reliability of subsystems with time

\begin{tabular}{c|c|c|c|c|c|c|c|c}
\hline Time (months) & $\mathbf{R}_{\mathbf{S 1}}(\mathbf{t})$ & $\mathbf{R}_{\mathbf{S 2}}(\mathbf{t})$ & $\mathbf{R}_{\mathbf{S 3} \mathbf{3}}(\mathbf{t})$ & $\mathbf{R}_{\mathbf{S 4}}(\mathbf{t})$ & $\mathbf{R}_{\mathbf{S 5}}(\mathbf{t})$ & $\mathbf{R}_{\mathbf{S 6}}(\mathbf{t})$ & $\mathbf{R}_{\mathbf{S 7}}(\mathbf{t})$ & $\mathbf{R}_{\mathbf{S y s}}(\mathbf{t})$ \\
\hline 0 & 1 & 1 & 1 & 1 & 1 & 1 & 1 & 1 \\
10 & 0.97044 & 0.92960 & 0.99104 & 0.88692 & 0.97531 & 0.95122 & 0.95599 & 0.70328 \\
20 & 0.94176 & 0.86415 & 0.98216 & 0.78662 & 0.95122 & 0.90483 & 0.91393 & 0.49460 \\
30 & 0.91393 & 0.80332 & 0.97336 & 0.69767 & 0.92774 & 0.860708 & 0.873716 & 0.34784 \\
40 & 0.88692 & 0.74676 & 0.96464 & 0.61878 & 0.904837 & 0.818731 & 0.835270 & 0.24463 \\
50 & 0.86070 & 0.69419 & 0.95599 & 0.54881 & 0.882497 & 0.778801 & 0.798516 & 0.17204 \\
60 & 0.83527 & 0.64532 & 0.94743 & 0.48675 & 0.860708 & 0.740818 & 0.763379 & 0.12099 \\
70 & 0.81058 & 0.59989 & 0.93894 & 0.43171 & 0.839457 & 0.704688 & 0.729789 & 0.08509 \\
80 & 0.78662 & 0.55766 & 0.93053 & 0.38289 & 0.818731 & 0.670320 & 0.697676 & 0.05984 \\
90 & 0.76337 & 0.51840 & 0.92219 & 0.33959 & 0.798516 & 0.637628 & 0.666977 & 0.04208 \\
100 & 0.74081 & 0.48190 & 0.91393 & 0.30119 & 0.77880 & 0.60653 & 0.63762 & 0.02959 \\
\hline
\end{tabular}

\section{System availability}

All seven subsystems are connected through one another in sequence. Just one failure leads to complete failure of the system. The overall system availability of the cooling tower is determined by

$$
\begin{aligned}
A_{S y s} & =A_{S_{1}} * A_{S_{2}} * A_{S_{3}} * A_{S_{4}} * A_{S_{5}} * A_{S_{6}} * A_{S_{7}} \\
& =0.9775468
\end{aligned}
$$

\section{System maintainability}

All seven subsystems are connected through one another in sequence. Just one failure leads to complete failure of the system. The overall system maintainability of the cooling tower is determined by

$$
\begin{aligned}
M_{S y s}(t) & =M_{S_{1}}(t) * M_{S_{2}}(t) * M_{S_{3}}(t) * M_{S_{4}}(t) * M_{S_{5}}(t) * M_{S_{6}}(t) * M_{S_{7}}(t) \\
= & \left(1-e^{-0.62 t}\right) *\left(1-e^{-1.25 t}\right) *\left(1-e^{-0.92 t}\right) *\left(1-e^{-4.48 t}\right) *\left(1-e^{-0.75 t}\right) *\left(1-e^{-0.95 t}\right) *\left(1-e^{-1.8 t}\right) \\
= & 1-e^{-4.0966 t}
\end{aligned}
$$

The variation in maintainability with respect to different time instant is compiled in table 3.

Table 3. Variation of maintainability of subsystems with time

\begin{tabular}{c|c|c|c|c|c|c|c|c}
\hline $\begin{array}{c}\text { Time } \\
\text { (months) }\end{array}$ & $\mathbf{M}_{\mathbf{S 1}}(\mathbf{t})$ & $\mathbf{M}_{\mathbf{S 2}}(\mathbf{t})$ & $\mathbf{M}_{\mathbf{S 3}}(\mathbf{t})$ & $\mathbf{M}_{\mathbf{S 4}}(\mathbf{t})$ & $\mathbf{M}_{\mathbf{S 5}}(\mathbf{t})$ & $\mathbf{M}_{\mathbf{S 6}}(\mathbf{t})$ & $\mathbf{M}_{\mathbf{S 7}}(\mathbf{t})$ & $\mathbf{M}_{\mathbf{S y s}}(\mathbf{t})$ \\
\hline 0 & 0.000000 & 0.000000 & 0.000000 & 0.000000 & 0.000000 & 0.000000 & 0.000000 & 0.000000 \\
10 & 0.997971 & 0.999996 & 0.999899 & 1.000000 & 0.999447 & 0.999925 & 1.000000 & 0.997239 \\
20 & 0.999996 & 1.000000 & 1.000000 & 1.000000 & 1.000000 & 1.000000 & 1.000000 & 0.999996 \\
30 & 1.000000 & 1.000000 & 1.000000 & 1.000000 & 1.000000 & 1.000000 & 1.000000 & 1.000000 \\
40 & 1.000000 & 1.000000 & 1.000000 & 1.000000 & 1.000000 & 1.000000 & 1.000000 & 1.000000 \\
50 & 1.000000 & 1.000000 & 1.000000 & 1.000000 & 1.000000 & 1.000000 & 1.000000 & 1.000000 \\
60 & 1.000000 & 1.000000 & 1.000000 & 1.000000 & 1.000000 & 1.000000 & 1.000000 & 1.000000 \\
70 & 1.000000 & 1.000000 & 1.000000 & 1.000000 & 1.000000 & 1.000000 & 1.000000 & 1.000000 \\
80 & 1.000000 & 1.000000 & 1.000000 & 1.000000 & 1.000000 & 1.000000 & 1.000000 & 1.000000 \\
90 & 1.000000 & 1.000000 & 1.000000 & 1.000000 & 1.000000 & 1.000000 & 1.000000 & 1.000000 \\
100 & 1.000000 & 1.000000 & 1.000000 & 1.000000 & 1.000000 & 1.000000 & 1.000000 & 1.000000 \\
\hline
\end{tabular}

\section{System dependability}

All seven subsystems are connected through one another in sequence. Just one failure leads to complete failure of the system. The overall system dependability of the cooling tower is determined by

$$
D_{\min (S y s)}=D_{\min \left(S_{1}\right)} * D_{\min \left(S_{2}\right)} * D_{\min \left(S_{3}\right)} * D_{\min \left(S_{4}\right)} * D_{\min \left(S_{5}\right)} * D_{\min \left(S_{6}\right)} * D_{\min \left(S_{7}\right)}
$$

$=0.977984582$

The summarized form of all the RAMD indices computed above for all the subsystems of cooling tower is given in table 4 which is as follows

Table 4. RAMD indices for cooling tower in STPP 


\begin{tabular}{|c|c|c|c|c|c|c|c|c|}
\hline RAMD indices & $\begin{array}{c}\text { Subsys } \\
\mathrm{S}_{1} \\
\end{array}$ & $\begin{array}{c}\text { Subsys } \\
\mathbf{S}_{2} \\
\end{array}$ & Subsys $\mathbf{S}_{\mathbf{3}}$ & Subsys $\mathbf{S}_{\mathbf{4}}$ & $\begin{array}{l}\text { Subsys } \\
S_{5} \\
\end{array}$ & $\begin{array}{c}\text { Subsys } \\
\mathbf{S}_{6} \\
\end{array}$ & $\begin{array}{c}\text { Subsys } \\
\mathrm{S}_{7} \\
\end{array}$ & System \\
\hline Reliability & $\mathrm{e}^{-0.003 \mathrm{t}}$ & $\mathrm{e}^{-0.0073 t}$ & $\mathrm{e}^{-0.0009 t}$ & $\mathrm{e}^{-0.012 \mathrm{t}}$ & $\mathrm{e}^{-0.0025 \mathrm{t}}$ & $\mathrm{e}^{-0.005 \mathrm{t}}$ & $\mathrm{e}^{-0.0045 \mathrm{t}}$ & $\mathrm{e}^{-0.0352 \mathrm{t}}$ \\
\hline Maintainability & $1-\mathrm{e}^{-0.62 t}$ & $1-\mathrm{e}^{-1.25 \mathrm{t}}$ & $1-\mathrm{e}^{-0.92 t}$ & $1-\mathrm{e}^{-4.48 \mathrm{t}}$ & $1-\mathrm{e}^{-0.75 t}$ & $1-\mathrm{e}^{-0.95 t}$ & $1-e^{-1.8 t}$ & $1-\mathrm{e}^{-4.0966 \mathrm{t}}$ \\
\hline Availability & 0.995185 & 0.994194 & 0.999023 & 0.999993 & 0.996678 & 0.994764 & 0.997506 & 0.977547 \\
\hline MTBF & 333.3333 & 136.9863 & 111.1111 & 83.3333 & 400.0000 & 200.0000 & 222.2222 & $\begin{array}{c}1486.986 \\
3\end{array}$ \\
\hline MTTR & 1.612903 & 0.800000 & 1.086957 & 0.000600 & 1.333333 & 1.052632 & 0.555556 & 6.441981 \\
\hline $\begin{array}{l}\text { Dependability } \\
\text { or Dmin }\end{array}$ & 0.995285 & 0.994334 & 0.999028 & 0.999993 & 0.996730 & 0.994881 & 0.997537 & 0.977985 \\
\hline $\begin{array}{l}\text { dependability } \\
\text { ratio }\end{array}$ & 206.6667 & 171.2329 & $\begin{array}{c}1022.222 \\
2\end{array}$ & $\begin{array}{c}138810.229 \\
2\end{array}$ & 300.0000 & 190.0000 & 400.0000 & \\
\hline
\end{tabular}

Table 5. Impact of failure rate of subsystems $S_{1} \& S_{2}$ on their reliability

\begin{tabular}{c|c|c|c|c}
\hline & \multicolumn{2}{|c|}{ System $\mathbf{S}_{\mathbf{1}}$} & \multicolumn{2}{c}{ Subsystem $\mathbf{S}_{\mathbf{2}}$} \\
\hline Time (in months) & $\boldsymbol{\beta}_{\mathbf{1}}=\mathbf{0 . 0 0 2}$ & $\boldsymbol{\beta}_{\mathbf{1}}=\mathbf{0 . 0 0 6}$ & $\boldsymbol{\beta}_{\mathbf{2}}=\mathbf{0 . 0 0 5}$ & $\boldsymbol{\beta}_{\mathbf{2}}=\mathbf{0 . 0 1 5}$ \\
\hline 0 & 1.000000 & 1.000000 & 1.000000 & 1.000000 \\
10 & 0.980199 & 0.941765 & 0.951229 & 0.860708 \\
30 & 0.960789 & 0.886920 & 0.904837 & 0.740818 \\
40 & 0.941765 & 0.835270 & 0.860708 & 0.637628 \\
50 & 0.923116 & 0.786628 & 0.818731 & 0.548812 \\
60 & 0.904837 & 0.740818 & 0.778801 & 0.472367 \\
70 & 0.886920 & 0.697676 & 0.740818 & 0.406570 \\
90 & 0.869358 & 0.657047 & 0.704688 & 0.349938 \\
100 & 0.852144 & 0.618783 & 0.670320 & 0.301194 \\
& 0.835270 & 0.582748 & 0.637628 & 0.259240 \\
\end{tabular}

Table 6. Impact of failure rate of subsystems $\mathrm{S}_{3} \& \mathrm{~S}_{4}$ on their reliability

\begin{tabular}{c|c|c|c|c}
\hline & \multicolumn{2}{|c|}{ Subsystem $\mathbf{S}_{\mathbf{3}}$} & \multicolumn{2}{c}{${\text { Subsystem } \mathbf{S}_{\mathbf{4}}}^{\boldsymbol{\beta}_{\mathbf{4}}=\mathbf{0 . 0 0 9}}$} \\
\hline Time (in months) & $\boldsymbol{\beta}_{\mathbf{3}}=\mathbf{0 . 0 0 0 2}$ & $\boldsymbol{\beta}_{\mathbf{3}}=\mathbf{0 . 0 0 7}$ & $\boldsymbol{\beta}_{\mathbf{4}}=\mathbf{0 . 0 0 1}$ & 1.000000 \\
10 & 1.000000 & 1.000000 & 1.000000 & 0.835270 \\
20 & 0.998002 & 0.932394 & 0.980199 & 0.697676 \\
30 & 0.996008 & 0.869358 & 0.960789 & 0.582748 \\
40 & 0.994018 & 0.810584 & 0.941765 & 0.486752 \\
50 & 0.992032 & 0.755784 & 0.923116 & 0.406570 \\
60 & 0.990050 & 0.704688 & 0.904837 & 0.339596 \\
70 & 0.988072 & 0.657047 & 0.886920 & 0.283654 \\
80 & 0.986098 & 0.612626 & 0.869358 & 0.236928 \\
90 & 0.984127 & 0.571209 & 0.852144 & 0.197899 \\
100 & 0.982161 & 0.532592 & 0.835270 & 0.165299 \\
\hline
\end{tabular}

Table 7. Impact of failure rate of subsystems $S_{5} \& S_{6}$ on their reliability

\begin{tabular}{c|c|c|c|c}
\hline & \multicolumn{2}{|c|}{ Subsystem $\mathbf{S}_{\mathbf{5}}$} & \multicolumn{2}{c}{${\text { Subsystem } \mathbf{S}_{\mathbf{6}}}^{\boldsymbol{\beta}_{\mathbf{6}}=\mathbf{0 . 0 0 9}}$} \\
\hline Time (in months) & $\boldsymbol{\beta}_{\mathbf{5}}=\mathbf{0 . 0 0 1 2}$ & $\boldsymbol{\beta}_{\mathbf{5}}=\mathbf{0 . 0 0 4 0}$ & $\boldsymbol{\beta}_{\mathbf{6}}=\mathbf{0 . 0 0 1}$ & 1.000000 \\
0 & 1.000000 & 1.000000 & 1.000000 & 0.913931 \\
20 & 0.988072 & 0.960789 & 0.990050 & 0.835270 \\
30 & 0.976286 & 0.923116 & 0.980199 & 0.763379 \\
40 & 0.964640 & 0.886920 & 0.970446 & 0.697676 \\
50 & 0.953134 & 0.852144 & 0.960789 & 0.637628 \\
60 & 0.941765 & 0.818731 & 0.951229 & 0.582748 \\
70 & 0.930531 & 0.786628 & 0.941765 & 0.532592 \\
80 & 0.919431 & 0.755784 & 0.932394 & 0.486752 \\
90 & 0.908464 & 0.726149 & 0.923116 & 0.444858 \\
100 & 0.897628 & 0.697676 & 0.913931 & 0.406570 \\
\hline
\end{tabular}




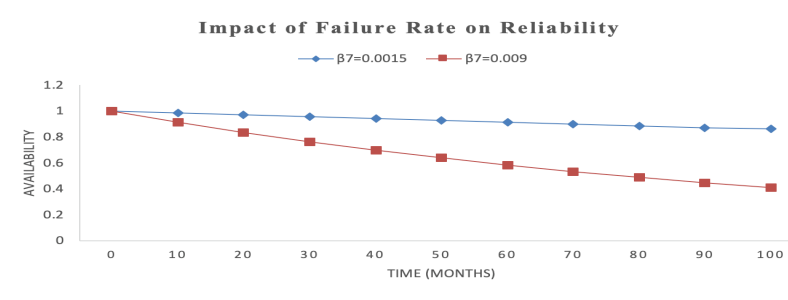

Fig 8. Effect of failure rate of subsystem $\mathrm{S}_{7}$ on subsystem's reliability Impact of Failure Rate on Reliability

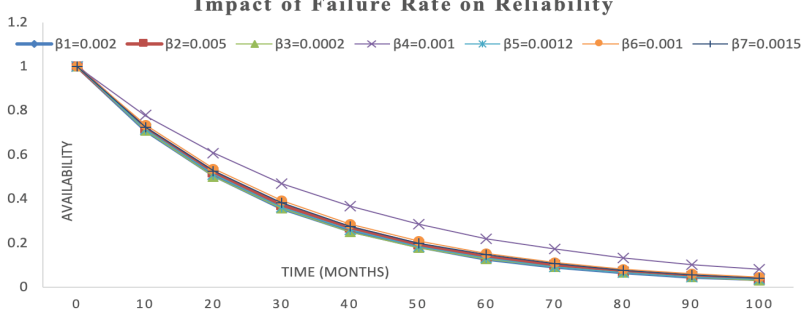

Fig. 9. Impact of failure rate of various subsystem's failure rate on system reliability

Impact of Failure Rate on Reliability

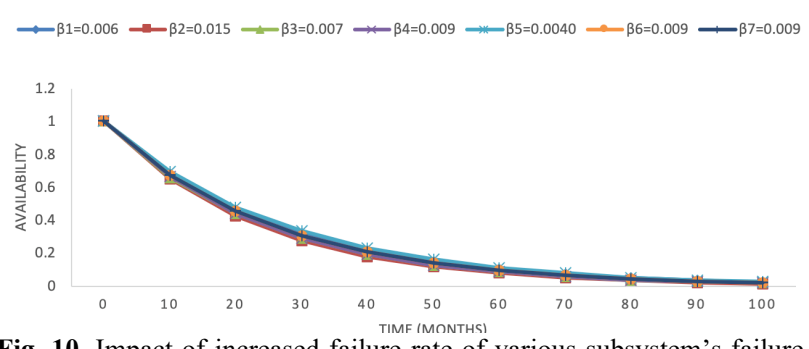

Fig. 10. Impact of increased failure rate of various subsystem's failure rate on system reliability

\section{Discussion and conclusion}

Empirical study for a particular case has been carried out by assigning numerical values to various parameters as given in Table 1 to obtain reliability measures of the various subsystems and system. Results for several subsystem's reliability and maintenance behaviors have been provided in Tables 2 and 3 respectively. Table 4 summaries all other RAMD measures. From the numerical analysis mentioned in table 2, it is revealed that after operation of 50 months cooling tower's reliability remains 0.172044864 only while automatic deaerator valves reliability is very low among all the subsystems and needs special attention. Hence system designers must plan some maintenance policy for it. From tables 5-7 and figures 3.8-3.10, it is revealed that as the failure rate increases the system's reliability sharply decreases. From this study, it is concluded that subsystem $\mathrm{S}_{4}$ i.e. deaerator valves are most critical and highly sensitive components and it require special attention to improve the reliability of the cooling tower. It is inferred that by monitor the failure rates of the deaerator valves and applying proper maintenance policies, management can improve the efficiency of the cooling tower and its working hours.

This is an Open Access article distributed under the terms of the Creative Commons Attribution License

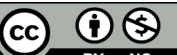

\section{References}

1) Casteren, J. F. L. Van, Bollen, M. H. J., and Schmieg, M. E. "Reliability assessment in electrical power systems: the WeibullMarkov stochastic model", IEEE Transactions on Industry Applications, 36(3): 2000, pp. 911-915.

2) Eti, M. C., Ogaji, S. O. T., and Probert, S. D. "Reliability of the Afam electric power generating station, Nigeria”, Applied Energy, 77(3), 2004, pp. 309-315.

3) Arora,N. and Kumar,D. "Availability analysis of steam and power generation systems in the thermal power plant", Microelectronics Reliability, 37(5), 1997, pp. 795-799.

4) Carazas,F.J.G. and Souza,G.F.M. "Availability Analysis of Gas Turbines Used in Power Plants", International Journal of Thermodynamics, 12 (1),2009, pp. 28-37.

5) Carazas,F.J.G., Salazar,C.H. and Souza,G.F.M. "Availability analysis of heat recovery steam generators used in thermal power plants", Energy, 36(6), 2009, pp. 3855-3870.

6) Kumari,R., Sharma,A.K. and Tewari,P.C. "Performance Evaluation of a Coal-fired Power Plant", International Journal of Performability Engineering, 9(4), 2013, pp.455-461.

7) Adhikarya,D.D., Bosea,G.K., Chattopadhyayb, S., Bosecand, D. and Mitra, S. "RAM investigation of coal-fired thermal power plants", International Journal of Industrial Engineering Computations, 3,2012, pp. 423-434.
8) Obeidat, S., Fouad, R. and Mandahawi, N. "Maintenance Management and Quality Improvement in AL-Hussein Thermal Power Station", Advances in Information Sciences and Service Sciences, 5(8), 2013, pp.18-26.

9) Aggarwal,K., Singh,V. and Kumar,S. "Availability analysis and performance optimization of a butter oil production system: a case study", International Journal of System Assurance Engineering and Management, 8(S1), 2014, pp. 538-554.

10. Corvaro, F., Giacchetta, G., Marchetti, B. and Recanati, M. "Reliability, availability, maintainability (RAM) study on reciprocating compressors", Petroleum, Vol. 3 No. 2, 2017, pp. 266272 .

11. Tsarouhas, P.. Reliability, availability and maintainability (RAM) analysis for wine packaging production line. International Journal of Quality \& Reliability Management, 35(3),2018, pp. 821-842.

12. Saini M, and Kumar A, "Performance analysis of evaporation system in sugar industry using RAMD analysis". J Braz Soc Mech Sci Eng 41:4, 2019.

13. Goyal, D., Kumar, A., Saini, M., and Joshi. H. "Reliability, maintainability and sensitivity analysis of physical processing unit of sewage treatment plant." SN Appl. Sci. 1: 1507, 2019. https://doi.org/10.1007/s42452-019-1544-7 


\section{List of notations and definitions}

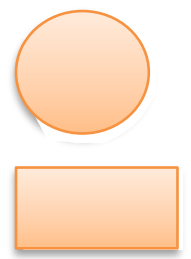

$\mathrm{A}, \mathrm{B}, \mathrm{C}, \mathrm{D}, \mathrm{E}$ and $\mathrm{F}$

$\mathrm{a}, \mathrm{b}, \mathrm{c}, \mathrm{d}$, e and $\mathrm{f}$

$\mathrm{D}_{1}$

$\beta_{i} i=1,2,3,4,5,6,7$

$\mu_{i} \quad i=1,2,3,4,5,6,7$

$p_{0}(t)$

$p_{i} ; i=0,1,2$

$f(x)=\left\langle\begin{array}{ll}\lambda e^{-\lambda x} ; 0 \leq x \leq \infty \\ 0 \quad ; \text { otherwise }\end{array}\right.$

$R(t)=P(T>t)=\int_{t}^{\infty} f(x) d x$

Availability function $=\frac{\text { Life time }}{\text { total time }}=\frac{\text { Life time }}{\text { Life time }+ \text { Repair time }}=\frac{M T T F}{M T T F+M T T R}$

$M(t)=P(T \leq t)=1-e^{\left(\frac{-t}{M T T R}\right)} \quad$ Maintainability function

$M T B F=\int_{0}^{\infty} R(t) d t=\int_{0}^{\infty} e^{-\theta t} d t=\frac{1}{\theta} \quad$ Mean Time Between Failures

$\operatorname{MTTR}=\frac{1}{\mu} \quad$ Mean Time to repair

$\mu=$ repair rate $; \quad \beta=$ failure rate

$d=\frac{\mu}{\beta}=\frac{M T B F}{M T T R}$

Dependability ratio

$D_{\min }=1-\left(\frac{1}{d-1}\right)\left(e^{-\operatorname{In} d / d-1}-e^{-d \operatorname{In} d / d-1}\right)$
System is in failure state

列

Repair rate of subsystems A, B, C, D, E, F and G respectively

Probability that system is in initial state with full capacity

Steady state probability that the system is in $i^{\text {th }}$ state

Probability density function of exponential distribution 